\title{
Screening and Identification of LAB for Degradation of Nitrite in Sichuan Paocai Water
}

\author{
Cao Hongfu' ${ }^{1}$ Qin Tao ${ }^{1}$, Zou Jinpeng ${ }^{1,2}$ and Shen Wenhao ${ }^{1}$ \\ ${ }^{1}$ College of Food Science, Sichuan Agricultural University, Ya'an, Sichuan, China \\ ${ }^{2}$ College of Agriculture, Sichuan Agricultural University, Chengdu, Sichuan, China
}

Keywords: Nitrite degradation, LAB, Screening, Identification

\begin{abstract}
Paocai is a traditional fermented food, which is loved by people because of its delicious. However, nitrite inevitably exists in Paocai, and excessive intake of nitrite will cause a certain degree of harm to the human body. Studies have shown that LAB have the ability to degrade nitrite, and $\mathrm{AD}$ and $\mathrm{ED}$ are the two main ways. In this experiment, $20 \mathrm{LAB}$ strains of that can degrade nitrite were screened from Paocai water. After $30 \mathrm{~h}$, the NDR of DAE was 97.69\% 99.71\%. L2 and L6 could degrade nitrite by high efficiency ED, which were screened by spectrophotometer. The two bacteria's NDR of DAE was $99.28 \%$ and $99.13 \%$, and the NDR of ED were respectively $90.89 \%$ and $90.02 \%$. The two strains were identified by molecular biology. The results showed that L2 was Lactobacillus plantarum and L6 Lactococcus lactis.
\end{abstract}

\section{Introduction}

Nitrite is a common nitrogenous compound[1], which can cause poisoning after excessive intake[2]. It can be usually found in meat, vegetable, water and so on[3]. Paocai is a traditional fermented food with a long history in Sichuan, China. After being cleaned, vegetables are put into Paocai water for a certain time to ferment, thus forming a unique taste and flavor[4]. In the process of fermentation, LAB plays an important role. LAB can produce lactic acid in the process of fermentation to improve Paocai acidity and bring unique flavor[5]. Not only that, LAB plays a regulatory role in human intestinal health to promote the digestion and absorption of human intestinal flora[6]. However, Paocai still contains nitrite. With the emergence of multiple nitrate poisoning events[7], people pay more attention to nitrite in Paocai. Therefore, many scholars are concerned about reducing nitrite in Paocai.

Many researches have been proved that LAB could degrade nitrite[8], and some scholars have made mixed fermentation and achieved remarkable results. Some Paocai were found to contain less nitrite through researchers directly add $\mathrm{LAB}[9]$. Zhou and others confirmed that inoculation of LAB fermentation can effectively reduce nitrite in food[10]. Kim have proved that most of the LAB have the function of degrading nitrite, those LAB degradation mechanism had been basically clear[11], $\mathrm{ED}$ at the early stage of fermentation, and $\mathrm{AD}$ at the later stage of fermentation[12].

At present, the NDR in the food field had become the hot problem[13]. Many studies had shown that LAB was beneficial to inhibit nitrite forming and reduce nitrite accumulation[14]. Therefore, this experiment used Paocai water as the material to screen LAB to degrade nitrite, meanwhile to stored bacteria resources for food industries.

\section{Materials and methods}

\section{Sampling}

Four home-made Paocai water were randomly sampled in Ya'an, sichuan province,which were kept at low temperatures and sent to the laboratory quickly.

\section{Medium}

MRS liquid medium: Peptone, glucose, potassium dihydrogen phosphate, yeast powder, diammonium citrate, sodium acetate, beef paste, manganese sulfate, TWAIN-80, magnesium sulfate, distilled water, 
MA: MRS medium, $2 \%$ calcium carbonate, $\mathrm{PH} 6.4$,

MSDN: MRS medium containing $125 \mathrm{mg} / \mathrm{L}$ sodium nitrite.

MSDNE: $125 \mathrm{mg} / \mathrm{L}$ sodium nitrite, MRS medium with $2 \%$ calcium carbonate, PH 6.4.

\section{Reagent}

Glacial acetic acid, zinc acetate, nitrite, sodium borate, ferrous cyanide potassium, anhydrous ethanol (AR), p-phenyl sulfonic acid, naphthalene ethyl hydrochloride two amine, etc. All reagents were analytically pure.

\section{Instrument and equipment}

Super-clean worktable, Ultra-low temperature refrigerator, spectrophotometer, optical microscope, high pressure sterilization pot, constant temperature biochemical culture box, electric constant temperature blast drying box and so on.

\section{Isolation and screening of LAB}

\section{Dilution of sample}

The $25 \mathrm{~mL}$ sample was added to the $225 \mathrm{~mL}$ saline, and then $1 \mathrm{~mL}$ of which was added to $9 \mathrm{ml}$ of saline. Repeat the operation until the sample is diluted to $10^{-8}$.

\section{Screening of LAB}

$10^{-4} \sim 10^{-8}$ samples respectively were cultured by pouring in MRS medium at $37{ }^{\circ} \mathrm{C}$ for $36 \sim 48$ $\mathrm{h}[15]$. The calcium dissoluting zone of LAB colony needed to be observed after the culture[16]. Referring to the bacterial identification manual, the single colony of suspected LAB was picked out from the cultured samples, and was inoculated on the MRS medium for screening bacteria $37{ }^{\circ} \mathrm{C}$. After $48 \mathrm{~h}$, single colony with marked calcium dissoluting zone were picked out for repeated culture to get LAB.

\section{Characteristics and microscopic examination of colonies}

The morphological characteristics of LAB were classified according to the manual of microbial identification[17].

Microscopic examination was observed with low magnification under ordinary optical microscope, and then using oil mirrors to observe the shape of LAB, which referred to the manual of microbial identification.

\section{Screening and identification of LAB that degraded nitrite \\ Preliminary screening}

Under the aseptic environment, the strains were activated in MA at $37{ }^{\circ} \mathrm{C}$ for $11 \sim 13 \mathrm{~h} .10 \mu \mathrm{L}$ bacteria solution was added to $10 \mathrm{~mL} \mathrm{MSDN}$ to be cultured at $\mathrm{pH} 6.4,30^{\circ} \mathrm{C}$, for $36 \sim 72 \mathrm{~h}$.

The samples were used to react with naphthalene ethylenediamine hydrochloric acid (chromogenic reaction: $2 \mathrm{~mL}$ p-aminophenol sulfonic acid first, after $5 \mathrm{~min}$, adding $1 \mathrm{~mL}$ naphthalene ethylenediamine solution, then observing and recording the color changes of each medium). Finally, the strains with lighter color were picked out as the strains screened preliminarily[18].

\section{Re-screening}

The strains came from preliminary screening was activated in MA for $12 \mathrm{~h} .60 \mathrm{~mL}$ of cultured MA was transferred to MSDN(containing $125 \mathrm{mg} / \mathrm{L} \mathrm{NaNO}$ and $0.4 \mathrm{~g} \mathrm{CaCO}_{3}$ ), $\mathrm{pH}$ at about 6.4, then all the samples were cultured in shaking bed at $100 \mathrm{r} / \mathrm{min}, 37{ }^{\circ} \mathrm{C}$. The weight of $\mathrm{NaNO}_{2}$ was conducted at intervals, and the NDR was calculated, judging by the spectrophotometer method in the chinese national standard GB5009.33-2016. At the same time, the standard curve and reagent blank were completed.

Reference to GB5009.33-2016 for measuring the weight of nitrite, according to the absorbance of different concentrations of the standard $\mathrm{NaNO}_{2}$ at $538 \mathrm{~nm}$, draw the standard curve about $\mathrm{A}_{\mathrm{NaNO} 2}$ $[\mathrm{L} / \mathrm{g} \cdot \mathrm{cm})]^{-} \mathrm{C}_{\mathrm{NaNO} 2}(\mathrm{mg} / \mathrm{kg})$.

The weight of nitrite (based on sodium nitrite) is calculated by formula based on GB5009.33-2016.

\section{Molecular sequence identification}

Strains were identified using $16 \mathrm{~S}$ rDNA gene sequencing. Omega's DNA extraction kit was used for total genomic DNA extraction, when strains grew to their late log phase. Fragments of bacterial 
16S rDNA were amplified by polymerase chain reaction (PCR) using the primers 27F (27F: 5'-AGAGTTTGATCCTGGCTCAG-3') and 1492R (5'-GGTTACCTTACGACTT-3') (Bioengineering Co., Inc., Shanghai, China) with the thermocycler. PCR amplification products were sequenced by Sanger chain termination method and spliced by Contig Express splicing program. The reaction parameters of PCR included five min of denaturation at $95^{\circ} \mathrm{C}$, followed by 35 cycles of $95^{\circ} \mathrm{C}$, for $30 \mathrm{~s}, 58^{\circ} \mathrm{C}$, for $30 \mathrm{~s}, 72^{\circ} \mathrm{C}$, for $90 \mathrm{~s}$, and a final extension at $72^{\circ} \mathrm{C}$, for $7 \mathrm{~min}$. After the reaction, the PCR products were subjected to $1 \%$ agarose gel electrophoresis to confirm the PCR amplification fragments. The purified PCR products were sent to Shanghai Bioengineering Limited by Share Ltd to sequence. Finally, the spliced sequence file was compared with the data in the NCBI ribosomal DNA sequence (Bacteria and Archaea) database by using the NCBI Blast program, and the strain information with the largest similarity to the sequence of the tested bacteria was obtained. In order to further display the relationship and system status of the tested strains and the known LAB, according to the results of the homologous sequence search, the ClustalX software was used to match the multiple sequences of the tested strains and the related strains (Alignment), and the booststrap method and neighbour-joining methods in the MEGA 6 biological software were used to construct phylogenetic tree with 1000 Bootstrap tests.

\section{Results and Analysis}

\section{Isolation and purification of LAB}

40 strains with obvious calcium dissolving zone were isolated from Paocai water. 36 strains of Gram-positive bacteria were observed by Gram staining, and LAB were generally chained or paired without spores.

\section{Preliminary screening of nitrite degrading strain}

The 36 screened strains were activated in MA for $12 \sim 14 \mathrm{~h}$, and then $10 \mu \mathrm{L}$ bacterial fluid was transferred to MSDN to incubate at $30^{\circ} \mathrm{C}$ for $42 \mathrm{~h}$. Subsequently, after the chromogenic reaction, 20 strains with light color were selected out.

\section{Rescreening of bacteria of degrading nitrite}

The nitrite degradation rate was measured by spectrophotometer in GB 5009.33-2016, 20 strains of bacteria were activated in MA, inoculated separately in MSDN and MSDNE, cultured at $30^{\circ} \mathrm{C}$ in the shaking bed, the weight of $\mathrm{NaNO}_{2}$ was detected after $6 \mathrm{~h}, 24 \mathrm{~h}$ and $36 \mathrm{~h}$ respectively, at the same time, reagent blank was set up to calculate nitrite degradation rate.

\section{The degradation rate of nitrite}

The nitrite degradation rate was measured according to the GB 5009.33-2016 spectrophotometer. The nitrite degradation rate of the 20 tested strains was obtained.

The results show that nitrite was firstly degraded by enzyme and then degraded by acid at the later stage of LAB fermentation. The NDR in all strains at $30 \mathrm{~h}$ could reach $97.69 \%$, and the NDR of the 20 LAB strains was stronger.

\section{The NDR by enzyme}

These strains was inoculated in MRS medium containing $\mathrm{NaNO}_{2}$ and $\mathrm{CaCO}_{3}$, shaking culture at $30^{\circ} \mathrm{C}, 100 \mathrm{r} / \mathrm{min}$, and the NDR of LAB were obtained.

Analyzing the data, we can conclude that at the early stage of LAB fermentation in Paocai water, the $\mathrm{pH}$ of the solution was about 6.4, and the nitrite was degraded through $\mathrm{ED}$, which was degraded by nitrite reductase produced by LAB. It could be seen from table 1 and table 2 that when the 20 strains of tested bacteria were at a higher $\mathrm{pH}$, their DNR decreased to some extent. However, it was obvious that the NDR of $\mathrm{L}_{2}$ and $\mathrm{L}_{6}$ could still reached more than $90 \%$ in a high $\mathrm{pH}$, so $\mathrm{L}_{2}$ and $\mathrm{L}_{6}$ had strong ability of ED.

\section{Identification of molecular sequence}

The DNA of $\mathrm{L}_{2}$ and $\mathrm{L}_{6}$ were extracted by DNA extraction kit,which were amplified by bacterial $16 \mathrm{~S}$ rDNA gene general primers to get $16 \mathrm{~S}$ rDNA product. The results of agarose gel electrophoresis were Figure 1. The size of the product is about $1500 \mathrm{BP}$, which was consistent with the standard material, indicating that the amplification is successful[19]. The PCR products were 
sent to Shanghai biological Co., Ltd. For sequencing, and the sequenced results were appended at the end of the article. The homology was compared with the sequence in Genbank, in the end, L2 was identified as Lactobacillus plantarum and $\mathrm{L}_{6}$ Lactococcus lactis. C1ustalX software was used to analyze multiple sequences of test strains and related strains (Alignment). The phylogenetic tree was constructed by using booststrap method and neighbour-joining methods in MEGA 6 biological software, and 1000 Bootstrap tests were performed, and the result was showed in Figure 2. L2 and L6 were clustered together, and their genetic relationships were very close, but they were far from Lactococcus lactis subspecies AB100803.1, Lactobacillus plantarum Argentina subspecies AJ640078.1, Lactobacillus plantarum KX057547.1, Wechsler AB023236.1 and AF111948.1.

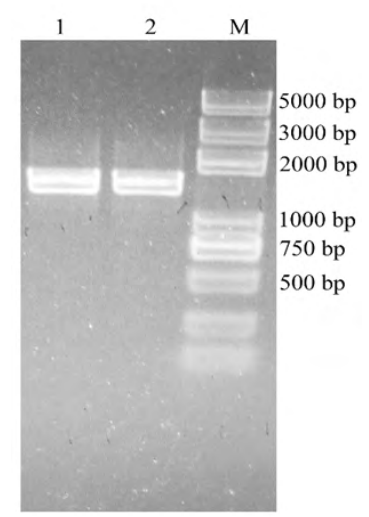

Figure 1 16S rDNA agarose gel electrophoresis of L2 and L6

M: DL 1500 DNA Marker, 1: $\mathrm{L}_{2}, 16 \mathrm{~S}$ rDNA, 2: $\mathrm{L}_{6}, 16 \mathrm{~S}$ rDNA

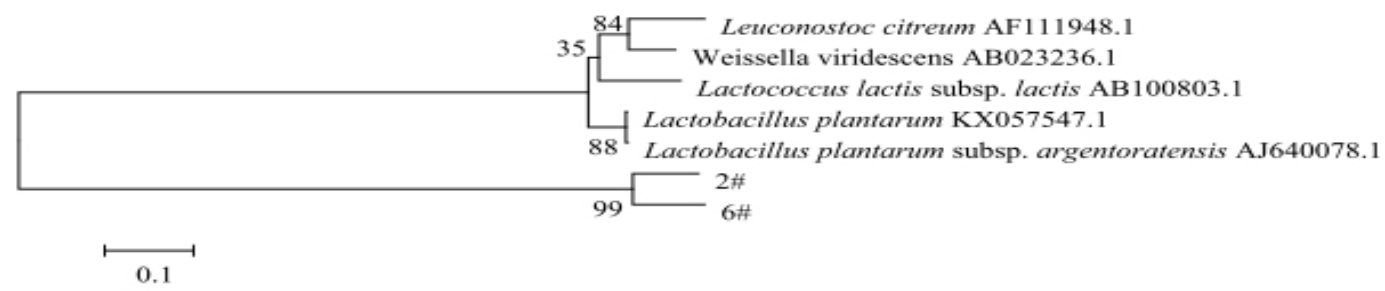

Figure 2 Phylogenetic tree of $\mathrm{L}_{2}$ and $\mathrm{L}_{6}$

\section{Discussion and Conclusion}

\section{Discussion}

\section{Time for nitrite reaction with $\mathrm{LAB}$}

According to the previous research, the experiment was conducted at $6 \mathrm{~h}, 24 \mathrm{~h}$ and $36 \mathrm{~h}$ asthe time point for measuring the degradation effect of nitrite.

The results of the pre experiment showed that the degradation rate of nitrite was little changed after $30 \mathrm{~h}$, and Hai Qing et al[20]directly detected the NDR after $24 \mathrm{~h}$. So in this experiment, the degradation rate was determined after 30 h.[21]

The ability of LAB to degrade nitrite in $6 \sim 24 \mathrm{~h}$ was not guaranteed to be the strongest. Therefore, the test of the NDR of $6 \mathrm{~h}, 24 \mathrm{~h}$ and $30 \mathrm{~h}$ was suitable for this experiment.

The results showed that, whether DOE or DOA, the NRD was not high after $6 \mathrm{~h}$. It was probably due to the low acidity at the early stage of fermentation, mainly depending on the DOE of LAB[22], and the growth of LAB was in the delay period, so the effect of the DON was not obvious.

\section{Methods for screening bacteria}

In this experiment, the preliminary screening was carried out first and then the rescreening, which was based on the experience of many scholars before.

Facts showed that bacteria with a weak ability to degrade nitrite could be largely removed by preliminary screening. But it could not be accurate, and the error was larger because it was observed by the naked eyes.

The rescreening could improve the accuracy of the measurement. In addition, preliminary 
screening could do better to relieve the work of rescreening. So preliminary screening firstly and then rescreening could not only ensure the accuracy of the results, but also improve the detection efficiency[23].

\section{Conclusion}

In China, the annual output of vegetables is high, but the preservation of vegetables has not been effectively solved, so many people make vegetables into Paocai water, and Paocai has a lot of beneficial effects on the human body and is widely favored by people. However, the excessive nitrite in Paocai has always been a hot issue. So in this experiment, 20 strains of LAB capable of degrading nitrite were screened out in Paocai water.

40 strains with the ability of dissolving calcium were isolated and purified from Paocai water, of which 36 were Gram-positive bacteria, and 20 strains of LAB that could degrade nitrite were screened at the end of preliminary screening.

DOE in the early stage of fermentation, DOA in later period, LAB degraded nitrite through the effect of nitrite reductase, $\mathrm{pH}$ 6.0 6.4, compared with nitrite degradation ability in the ordinary screening medium without acidity control, 20 strains found in the preliminary screening could degrade nitrite obviously during the fermentation process. After $30 \mathrm{~h}$ fermentation, the NDR can reach $97 \%$.

When the $\mathrm{pH}$ was controlled between 6.0 6.4, the LAB degraded nitrite through DOE, and the NDR of the 20 strains generally decreased, but the $\mathrm{L}_{2}$ and $\mathrm{L}_{6}$ still had high NDR. The NDR of $\mathrm{L}_{2}$ reached $99.28 \%$ after $30 \mathrm{~h}$, the NDR by enzyme was $90.89 \%$. The NDR by enzyme of $\mathrm{L}_{6}$ was $90.02 \%$. Therefore, it could be concluded that nitrite reductase of $\mathrm{L}_{2}$ and $\mathrm{L}_{6}$ had obvious effect and enzyme degradation ability was better than other strains.

\section{References}

[1] Cisse IA, Mao X. Nitrate: Health Effect in Drinking Water and Management for Water Quality [J]. Environmental Research Journal.2008(6): 311-316.

[2] Shapiro L, Blackie H, Arthur D, Ross J, Eason C. Secondary poisoning risk for encapsulated sodium nitrite, a new tool for possum control [J]. New Zealand Journal of Ecology. 2018.

[3] Ikeda T. Effects and Problems of Nitrate and Nitrite Water Pollution Control Measures and Future Course of Necessary Measures [J]. Journal of Hydrology and Water Resource. 2012, 25(3): 163-173.

[4] Wang Z, Shao Y. Effects of microbial diversity on nitrite concentration in pao cai, a naturally fermented cabbage product from China [J]. Food Microbiology 2018, 72: 185-92.

[5] Yan PM, Xue WT, Tan SS, Zhang H, Chang XH. Effect of inoculating lactic acid bacteria starter cultures on the nitrite concentration of fermenting Chinese paocai $[\mathrm{J}]$. Food Control. 2008, 19(1): 50-55.

[6] Sorochan W. and friendly bacteria in the large intestine are essential to good health [M]. 2013.

[7] Luo H, Peng MQ, Zhang XD, Shi J. A Case Report on Food Poisoning Caused by Eating Nitrite by Mistake [J]. Chinese Journal of Food Hygiene. 2010.

[8] Yu SM, Zhang Y. Effects of Lactic Acid Bacteria on Nitrite Degradation during Pickle Fermentation [J]. Advanced Materials Research 2013, 781-784: 1656-60.

[9] Esmaeilzadeh P, Darvishi S, Ebrahimi K, Mirahmadi F, Vaziri M. Consideration of Lactic Acid Bacteria Ability to Reduce Nitrite Concentration in Standard De Man, Rogosa and Sharpe (Mrs) Broth-Sodium Nitrite Medium During Fermentation Period [J]. World Applied Sciences Journal 2012, 18(3).

[10] Zou LG, Yuan-Feng WU, Zhao Y. Study on change of nitrite content during vegetable fermentation by lactic acid bacteria [J]. Food Science \& Technology. 2006.

[11] Kim J, Chun J, Han HU. Leuconostoc kimchii sp. nov., a new species from kimchi [J]. International Journal of Systematic \& Evolutionary Microbiology. 2000, 50(5): 1915-19. 
[12] Zhang Q. The Study on Mechanism of Nitrite Degradation by Lactic Acid Bacteria [J]. Food \& Fermentation Industries. 2002.

[13] Yang XM, Liu QM, Xi yuan XU, Tang AZ. Effects of fermentation inoculated lactobacillus on quality and nitrite content of Chinese sauerkraut [J]. Journal of Zhejiang Agricultural University. 2003.

[14] Yuan Y, Chi JY, Huang DD, Zhao SS, Zhang QX, Chen W. Effect of lactic acid bacteria inoculated on sensory quality and nitrite concentration of pickles $[\mathrm{J}]$. Science \& Technology of Food Industry 2012, 33(7): 119-121.

[15] Yi YU, Qin-Feng QU, Xue-Lian HU. The Result Differences by Pouring Method and Coating Method in Detecting Content of Lactic Acid Bacteria in Food [J]. Academic Periodical of Farm Products Processing. 2012.

[16] Zhu J, Zhao J, Zhu Y. Isolation and identification of nitrite-degrading lactic acid bacteria from natural fermented vegetable [J]. China Brewing. 2015.

[17] Sandle T. 9 - Microbial identification. In: Sandle T, editor. Pharmaceutical Microbiology [J]. Oxford: Woodhead Publishing, 2016. 103-113.

[18] Gaya UI, Alimi S. Spectrophotometric determination of nitrate in vegetables using phenol [J]. Journal of Applied Sciences \& Environmental Management. 2006, 10(1).

[19] Olatunde OO, Obadina AO, Omemu AM, Oyewole OB, Olugbile A, Olukomaiya OO. Screening and molecular identification of potential probiotic lactic acid bacteria in effluents generated during ogi production [J]. Annals of Microbiology. 2018: 1-11.

[20] Hai-Qiang LU, Huo WM, Xin-Xi GU, Chen LI, Tian HT. Isolation and characterization of psychrotrophic lactic acid bacteria producing nitrite reductase [J]. Journal of Agricultural University of Hebei. 2015.

[21] Dodds KL, Collins-Thompson DL. Nitrite tolerance and nitrite reduction in lactic acid bacteria associated with cured meat products [J]. International Journal of Food Microbiology 1984, 1(3): 163-170.

[22] Xiao-Jing SU, Wen-Tao LI, Chen P, Yu-Kuo LI, Wang P. Preparation and Degradation Characteristics of Freeze-dried Lactic Acid Bacteria with Efficient Degradation of Nitrite [J]. Academic Periodical of Farm Products Processing. 2014.

[23] Pei H, Jiang H, Min Z. Screening and identification of lactic acid bacteria degrading nitrite [J]. China Brewing. 2013.

\section{Abbreviation in this article}

LAB: lactic acid bacteria, AD: acid degradation, ED: enzyme degradation, NDR: nitrite degradation rate, DAE: degradation of acid and enzyme, MRS: De'Man Rogosa Sharpe, MA: medium for activating strains, MSDN: medium for screening strains that degrade nitrite, MSDNE: medium for screening strains that degrade nitrite by enzyme 